\title{
El perfil del periodista jurídico actual en España
}

\author{
María José ANTich GiL \\ antich.marajos@gmail.com \\ Universidad CEU - Cardenal Herrera
}

Recibido: 7 de febrero de 2014

Aceptado: 9 de septiembre de 2014

\section{Resumen}

En este artículo se analizan los resultados obtenidos de una serie de entrevistas en forma de cuestionarios enviados a profesionales de la información jurídica, que pertenecen a diferentes medios de comunicación, tanto de prensa como de audiovisuales, con el fin de obtener un perfil del periodista jurídico actual en España.

Con la aplicación del análisis de contenido a la entrevista se pretende crear este perfil mediante una serie de características, tales como la edad, estudios, posgrado, especialidad y experiencia.

Palabras clave: periodista jurídico, perfil profesional, análisis de contenido, entrevista.

\section{The Profile of the Current Legal Journalist in Spain}

\begin{abstract}
This article discusses the results obtained from a series of interviews in the form of questionnaires sent to professionals in the legal information, which belong to different means of communication, both for press and media, in order to obtain a profile of the current legal journalist in Spain.

With the application of content analysis to the interview is intended to create this profile through a series of features, such as age, studies, postgraduate studies, specialty and experience.

Keywords: legal journalist, professional profile, content analysis, interview.

\section{Referencia normalizada}

ANTICH GIL, María José (2015): "El perfil del periodista jurídico actual en España". Estudios sobre el Mensaje Periodístico. Vol. 21, Núm. 1 (enero-junio), págs.: 243-249. Madrid, Servicio de Publicaciones de la Universidad Complutense.
\end{abstract}

Sumario: 1. Introducción. 2. Objetivos e hipótesis. 3. Metodología. 4. Resultados. 5. Conclusiones. 6. Referencias bibliográficas. 7. Anexos.

\section{Introducción}

La información jurídica es una realidad presente en la actualidad, puesto que todos los días aparecen noticias vinculadas al mundo del Derecho, las leyes, los tribunales, etc. El gran volumen de este tipo de información hace plantearnos qué formación tienen los profesionales que se dedican a ello, así como qué materias jurídicas son su especialidad o cuántos años llevan dedicados a esta profesión. El tratamiento de esta información así como la utilización de su lenguaje, los tecnicismos jurídicos que se emplean, nos llevan a querer crear un perfil del periodista jurídico actual, saber quién nos informa.

Por periodista entendemos que es aquella persona que ha realizado los estudios de Ciencias de la Comunicación o aquella persona que proviene de otras enseñanzas y que se ha formado con un posgrado en comunicación para ejercer el periodismo. Por tanto, su actividad principal es ofrecer información mediante cualquier soporte informativo, ya sea prensa, radio, televisión o internet. Por tanto, podemos deducir que periodista jurídico es aquel cuya especialidad es informar sobre todos los ámbitos de la justicia, los tribunales, los sucesos, las sentencias, las leyes y los delitos. Además el 
periodista jurídico tiene una buena formación en el área del Derecho, puesto que domina el lenguaje jurídico y puede trabajar tanto en la prensa generalista como en la especializada, donde se exige mayor rigor de especialización.

El periodista jurídico no necesariamente se vincula a la sección de tribunales sino que puede trabajar en cualquier área donde se traten todos los ámbitos citados. Así pues un periodista jurídico puede cubrir la información política, económica, de sucesos, de tribunales, la dedicada a famosos, etc.

\section{Objetivos e hipótesis}

Existen dos objetivos claves para esta investigación:

- Preparar una serie de cuestionarios para averiguar cuál es la edad, estudios, medio en el que trabaja y rama o especialidad de la información jurídica a la que se dedica o de la que es especialista.

- Crear un perfil de periodista jurídico actual mediante el cuestionario realizado.

En cuanto a las hipótesis, nos planteamos las siguientes:

- Nos planteamos si el periodista jurídico es una persona de edad madura, puesto que se necesita años de experiencia y formación para ejercer este tipo de periodismo, o por el contrario es una persona muy joven e inexperta a la hora de tratar temas jurídicos.

- Se pretende comprobar si los periodistas jurídicos son periodistas o tienen otra clase de estudios como Derecho, Economía, etc., si tienen formación de posgrado complementaria a sus estudios universitarios, etc.

- También, si ejercen una única especialidad en este ámbito (civil, penal, administrativo, etc.) o se mueven en todas las especialidades porque la información lo requiere. Así como saber los años de experiencia que tienen, cuánto tiempo llevan dedicándose a ejercer este tipo de periodismo.

\section{Metodología}

La metodología que hemos empleado es la del análisis de contenido aplicado a la entrevista, es decir, el análisis de contenido por racimo, donde "se pretende el establecimiento final de un conjunto o racimo de proposiciones, a partir de las cuales, se podrá reconstruir el discurso". Esta modalidad de análisis "permite sobrepasar el simple establecimiento de los temas principales y su frecuencia, siendo muy amplias las posibilidades en lo concerniente a la profundidad del estudio y al consecuente descubrimiento de aspectos subyacentes en el mismo" (Bermúdez, 1986:135-143).

Para llevar a cabo la efectividad de este análisis se han realizado unas entrevistas a través de un cuestionario, que ha sido expuesto a diferentes profesionales del mundo del periodismo jurídico y que pertenece a diferentes medios de comunicación, tanto de prensa escrita como audiovisuales. Se ha querido utilizar el cuestionario escrito para que las respuestas pudieran ser suficientemente razonadas. La mayor parte de los cuestionarios enviados y respondidos provienen de los miembros de la Asociación de Comunicadores e Informadores Jurídicos (ACIJUR). Además, también se ha enviado a otros profesionales que no pertenecen a la asociación pero que ejercen como periodistas jurídicos en diferentes medios de comunicación. 
Con este cuestionario se pretende crear un perfil del profesional jurídico en la actualidad. En total casi un cincuenta y seis por ciento de los profesionales han contestado al cuestionario enviado, respondiendo a las preguntas referentes a la edad, sexo, estudios de licenciatura, estudios de posgrado, idiomas, materia en la que se trabaja (civil, penal, administrativa, etc.) y años de experiencia.

\section{Resultados}

De los cuestionarios enviados más de la mitad han contestado a las preguntas realizadas. Los profesionales del periodismo jurídico, cuyas edades comprendidas entre cuarenta y cincuenta años son los que ocupan un mayor porcentaje en el gráfico, un 36 por ciento. El intervalo de treinta a cuarenta le sigue con un 32 por ciento, los que se sitúan entre cincuenta y sesenta años son un 16 por ciento. Con un porcentaje menor del 12 por ciento, se encuentran aquellos que tienen más de sesenta años y los menores de treinta años con un cuatro por ciento.

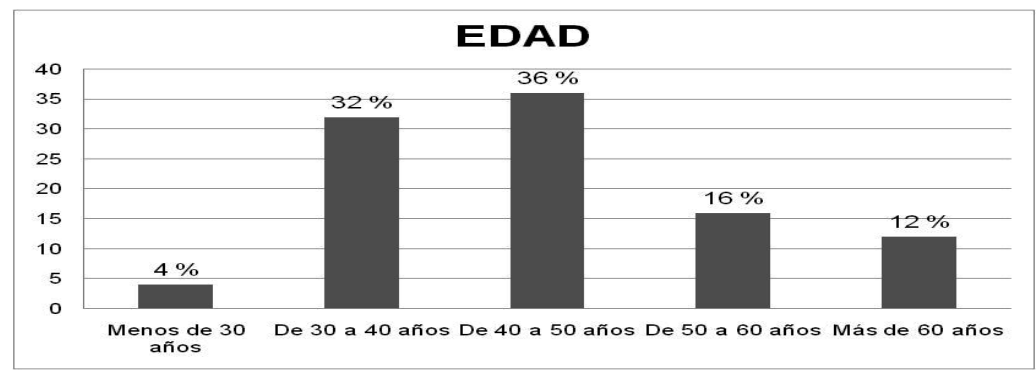

Gráfico 1. Edad del periodista jurídico. Elaboración propia.

Además, se puede destacar que de estos cuestionarios respondidos más de la mitad de los profesionales son hombres con un 52 por ciento, mientras que un 48 por ciento son mujeres. Los expertos en comunicación que han contestado el cuestionario tienen todos estudios superiores, es decir, titulación universitaria. Como profesionales del periodismo jurídico pensamos que las carreras universitarias que tendrían principalmente serían Periodismo o Derecho, o ambas. Pero nos hemos encontrado con gente que tiene Periodismo y otras carreras que no tienen que ver con Derecho, aunque son una minoría, como también lo son los profesionales que han estudiado Derecho con otras carreras que no tienen relación ninguna con el periodismo.

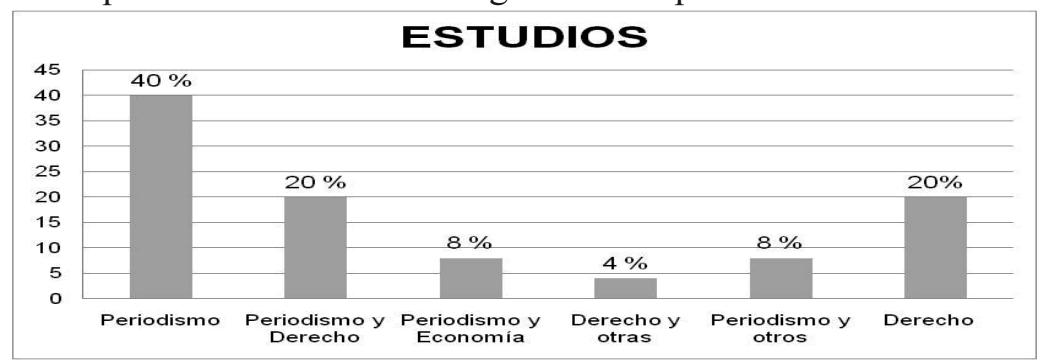

Gráfico 2. Estudios universitarios del periodista jurídico. Elaboración propia. 
La mayoría de estos profesionales, un 40 por ciento, tienen estudios de Periodismo. Les siguen los que tienen formación en Periodismo y Derecho, y los que tienen solo Derecho, con un 20 por ciento cada uno. Con un ocho por ciento están los licenciados en Periodismo y Economía y en Periodismo y otros. Por último, con un cuatro por ciento, están aquellos que han estudiado Derecho y otra carrera. Entre estas "otras carreras" está principalmente Ciencias Políticas, le siguen también Historia, Literatura, Filosofía, etc., pero estas últimas son menos frecuentes.

Dentro de los estudios nos interesaba saber si los profesionales del periodismo jurídico tienen estudios de posgrado, esto es si han realizado algún máster o doctorado, o ambas cosas. Un 48 por ciento no tienen estudios de posgrado. Un 32 por ciento han realizado máster, un 12 por ciento máster y doctorado. Por último, un ocho por ciento tienen estudios de doctorado.

Cabe destacar que los que han contestado que tienen estudios de máster y doctorado no significa que hayan hecho la tesis doctoral, sino que tienen realizados los cursos de doctorado o el Diploma de Estudios Avanzados (DEA). Por el contrario, los que sí que tienen el doctorado implica que han finalizado sus estudios con la realización de la tesis doctoral.

Los idiomas son muy importantes hoy en día para cualquier profesión. En este caso hemos establecido el inglés como único idioma principal, el inglés y francés, el inglés con otros idiomas, solo francés y ninguno o solo español, ya que hemos considerado que el español, catalán, gallego, etc., son considerados lengua materna y no representan ninguna aportación. El francés se ha incluido porque de normal suele ser el segundo idioma que se habla después del inglés que es el principal.

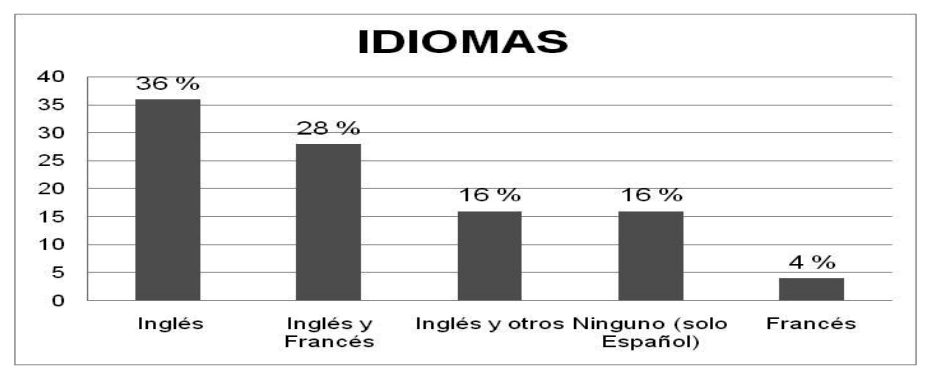

Gráfico 3. Idiomas que domina el periodista jurídico. Elaboración propia.

Los profesionales que hablan inglés son un 36 por ciento. Con un 28 por ciento están los que hablan inglés y francés. Se encuentran con el mismo porcentaje, un 16 por ciento, los que hablan inglés y otro idioma o idiomas (que no es el francés), y los que no hablan ninguno, es decir, que solo hablan el español que es su lengua materna. Entre los otros idiomas que no son inglés ni francés se encuentra el alemán. Por último, un cuatro por ciento habla el francés solamente.

En cuanto a la especialidad en la que se trabaja nos referimos a la materia, es decir, si es derecho civil, penal, administrativo, etc. En este caso, y basándonos en los cuestionarios contestados, nos hemos encontrado con profesionales que tratan todas las materias en general, con aquellos que solo trabajan en la parte de penal y civil, aque- 
llos que se ocupan de penal, civil y administrativo, los que se ocupan de otras materias no establecidas en el cuestionario como puede ser mercantil, y por último, aquellos que no han contestado a este punto del cuestionario.

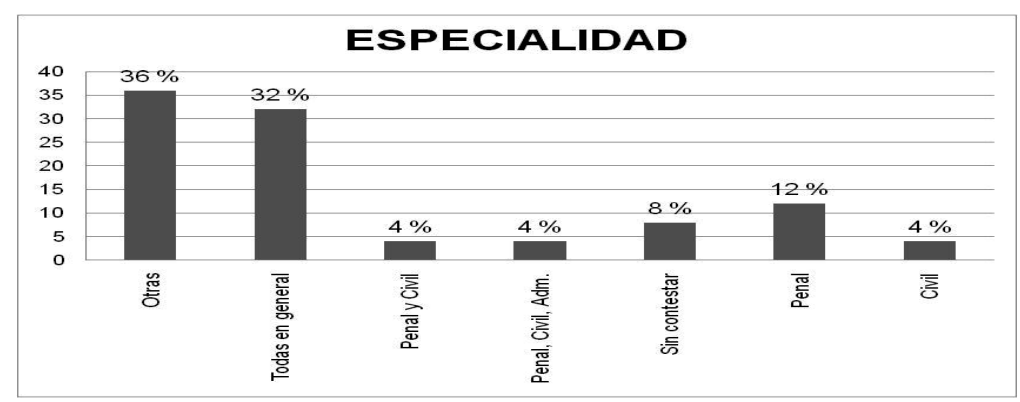

Gráfico 4. Especialidad en la que trabaja el periodista jurídico. Elaboración propia.

Los profesionales que se dedican a otras materias son un 36 por ciento. Entre estas otras están el derecho mercantil, laboral, derecho de marketing y de nuevas tecnologías, la gestión de despachos de abogados, todo lo relacionado con cuestiones institucionales en un despacho de comunicación, periodismo jurídico divulgativo, comunicación corporativa aplicada al mundo jurídico, política de Justicia, Derecho de la Unión Europea, derecho constitucional, seguridad jurídica preventiva, etc. Se entiende que los profesionales que trabajan en un despacho de abogados tocan todas las materias en general, que en este caso son un 32 por ciento. Los que se dedican exclusivamente al derecho penal son un 12 por ciento, y un cuatro por ciento se dedican al derecho civil, a penal y civil o a penal, civil y administrativo.

Por otro lado, un ocho por ciento no han contestado a esta pregunta del cuestionario. La experiencia de trabajo que aporta el profesional es importante. Se han establecido los siguientes intervalos: profesionales con menos de diez años de experiencia, de diez a veinte años, de veinte a treinta años, y más de treinta años de experiencia.

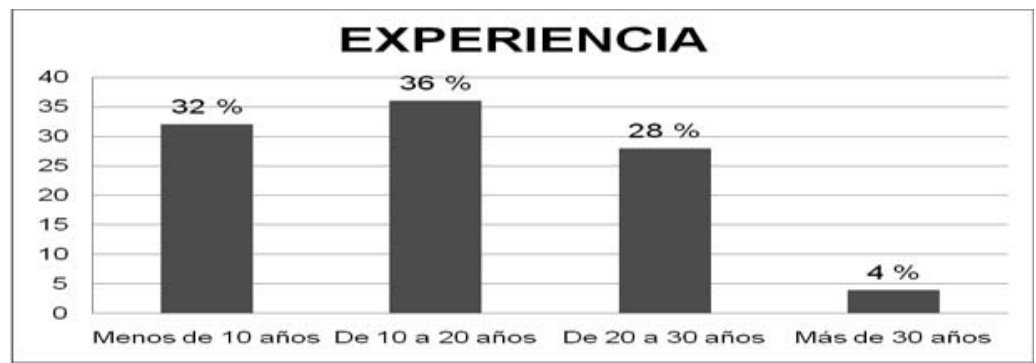

Gráfico 5. Experiencia del profesional jurídico. Elaboración propia.

De los parámetros representados se deduce que un 32 por ciento tienen una experiencia de menos de diez años, un 36 por ciento de diez a veinte años, un 28 por ciento lleva trabajando de veinte a treinta años. Por último, con un cuatro por ciento, están los que han trabajado más de treinta años y todavía continúan. 


\section{Conclusiones}

De todo el análisis se concluye que el perfil del periodista jurídico en la actualidad está representado por profesionales que tienen una edad comprendida entre los cuarenta y cincuenta años, y que más de la mitad son hombres.

Según los datos obtenidos se observa que un 36 por ciento tiene estudios de Periodismo y otra carrera como Derecho o Economía, un 52 por ciento tiene estudios de posgrado y un 44 por ciento habla dos o tres idiomas, incluyendo en ellos el español, inglés y otro idioma como el francés o el alemán.

En cuanto a la especialidad jurídica a la que se dedican, los porcentajes están muy repartidos, pero destacan aquellos que trabajan con todas las materias en general (penal, civil, administrativo, etc.), y aquellos que se dedican a otras, tales como derecho mercantil, derecho constitucional, etc.

La experiencia profesional con la que cuenta actualmente el periodista jurídico se sitúa entre menos de diez y hasta veinte años.

\section{Referencias bibliográficas}

BERMÚDEZ, Marlen (1986): "Aplicación del análisis de contenido a la entrevista". Ciencias Sociales, $\mathrm{n}^{\circ}$. 33. Universidad de Costa Rica, pp. 135-143.

\section{Anexos}

A continuación se muestra el modelo de cuestionario enviado a los diferentes profesionales del periodismo jurídico.

\section{a. Edad}

$\ulcorner$ Menos de 30 años

$\sqsubset$ De 30 a 40 años

$\sqsubset$ De 40 a 50 años

$\ulcorner$ De 50 a 60 años

$\ulcorner$ Más de 60 años

\section{b. Sexo}

ᄃ Masculino

$\sqsubset$ Femenino

\section{c. Estudios}

$\ulcorner$ Periodismo

$\sqsubset$ Derecho

$\sqsubset$ Economía

Г Otros (indicar cuáles) 


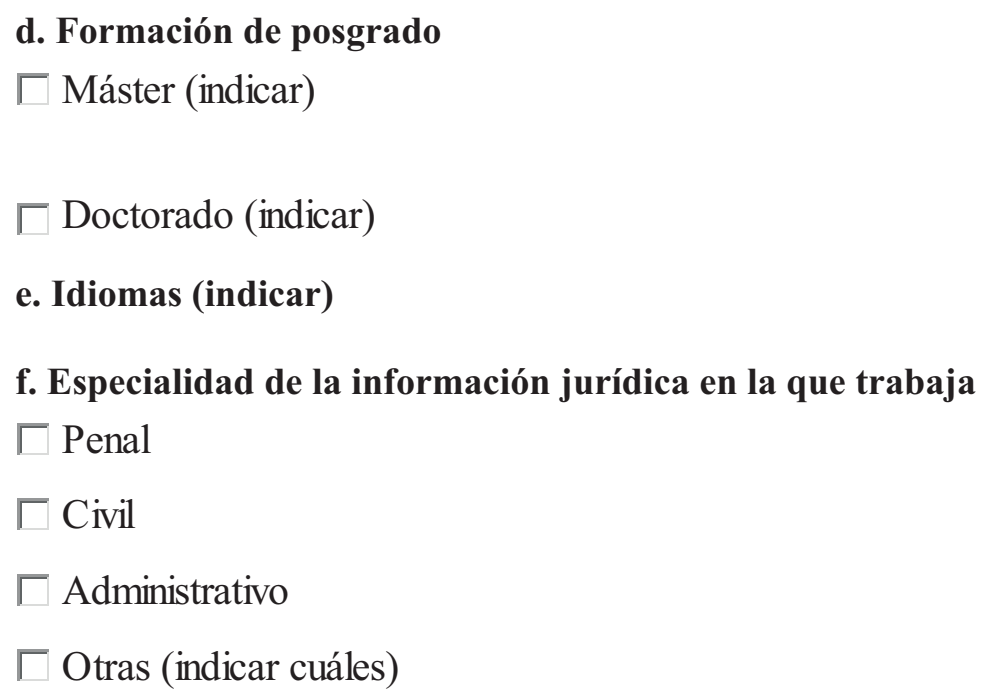

g. Años de experiencia como periodista jurídico

$\ulcorner$ Menos de 10 años

$\ulcorner$ De 10 a 20 años

$\sqsubset$ De 20 a 30 años

$\ulcorner$ Más de 30 años

GRACIAS POR SU COLABORACIÓN 